\title{
Analysing Cyber-resiliency of a Marine Navigation System using Behavioural Relations
}

Nissov, Morten C.; Dagdilelis, Dimitrios; Galeazzi, Roberto; Blanke, Mogens

Published in:

Proceedings of 2021 European Control Conference

Link to article, DOI:

10.23919/ECC54610.2021.9654972

Publication date:

2021

Document Version

Peer reviewed version

Link back to DTU Orbit

Citation (APA):

Nissov, M. C., Dagdilelis, D., Galeazzi, R., \& Blanke, M. (2021). Analysing Cyber-resiliency of a Marine Navigation System using Behavioural Relations. In Proceedings of 2021 European Control Conference (pp. 1385-1392). IEEE. https://doi.org/10.23919/ECC54610.2021.9654972

\section{General rights}

Copyright and moral rights for the publications made accessible in the public portal are retained by the authors and/or other copyright owners and it is a condition of accessing publications that users recognise and abide by the legal requirements associated with these rights.

- Users may download and print one copy of any publication from the public portal for the purpose of private study or research.

- You may not further distribute the material or use it for any profit-making activity or commercial gain

- You may freely distribute the URL identifying the publication in the public portal 


\title{
Analysing Cyber-resiliency of a Marine Navigation System using Behavioural Relations
}

\author{
Morten C. Nissov ${ }^{1}$, Dimitrios Dagdilelis ${ }^{1}$, Roberto Galeazzi ${ }^{1}$, and Mogens Blanke ${ }^{1}$
}

\begin{abstract}
Marine vessels need trustworthy navigation data for safe manoeuvring, but threats exist for external manipulation of signals and on-board systems. This paper employs analysis of behaviours to cross-validate that instruments provide correct information. Deviations from normal behaviour could be effects of malicious cyber-attack or instrument malfunction. Independent of the root cause, faulty information need be disregarded for navigation. This paper shows how instruments' violation of correct behaviour can be detected and isolated during near-coast navigation. The approach is to analyse topology of information flow and information processing, also referred to as structural analysis. The paper addresses the diagnosis potential for isolation of erroneous information about state of own ship and of surrounding objects. The analysis includes position, ship speed, and heading, which could lead to errors in navigation, to collision or grounding. The paper addresses required sensors, according to the International Maritime Organizations (IMO) Safety of Life at Sea (SOLAS) [20], and also presents potential gains by inclusion of computer vision. Showing that all single and several cases of simultaneous defects are discovered, for own ship and in surroundings, the results demonstrate that resilience of navigation information can be obtained for vessels sailing in coastal waters.
\end{abstract}

\section{INTRODUCTION}

Trustworthy and accurate sensor information is crucial for the operation of a vessel, even more so for vessels operating with limited to zero reliance on human intervention. As a result unintended faults or cyber-attacks in marine navigation systems pose a great risk to the ability of the vessel to operate safely, if at all. Typically, the challenge of identifying an either faulty or spoofed sensor has been left to the navigators. This creates reliance on human operators to notice and compensate for such malfunctions in real-time, where in coastal navigation the time horizon for reacting to potentially dangerous outcomes can be short. This problem becomes debilitating with respect to vessels striving for higher levels of autonomy as here the vessel must function as expected with minimal or potentially no human intervention, leaving identification and handling of erroneous sensor information up to the on-board navigation system. Vulnerabilities to cyber-attacks have been shown for the Automatic Identification System (AIS) [1]; the Electronic Chart and Display Information System (ECDIS) [2], [3]; the radar system [4]; the Global Navigation Satellite System (GNSS) [5].

\footnotetext{
${ }^{1}$ Department of Electrical Engineering, Technical University of Denmark, Kgs. Lyngby, DK 2800 morten.c.nissovegmail.com; \{dimda, rg, mb\}elektro.dtu.dk

This work was supported by the SAFENAV research project conducted for the Danish Maritime Authority and sponsored by the Danish Maritime Fund. Grant number is: 2018-136.
}

Investigation into methods and tools for detection of cyberattacks in navigation sensors has attracted significant interest and solutions exist that provide excellent first line of defence against intruders. The second line of defence is onboard, making all individual systems resistant to attacks. This is a difficult exercise on new vessels and hardly achievable on existing fleet. Jamming and spoofing of GNSS provided position represent the most threatening scenario since such system is the primary source of position information. The GNSS system's vulnerability to cyber-threats was demonstrated for both simple and complex attack strategies in [6], [7], and [8]. Effects of spoofing in sensor fused systems was shown by [9]. The capture of an UAV by GNSS spoofing was presented in [10]. Several spoofing and detection strategies were compared in [11], including pseudo-range monitoring, fusion with IMU, and physical antenna setup changes. Detection of attack on GNSS fused with IMU was addressed in [12].

The adoption of multi-sensor navigation systems as a mean to increase the GNSS resiliency was advocated in [13] and increased resiliency through information fusion was presented in [14], where AIS information and radar measurements from land were fused to monitor AIS spoofed positions. However, the real-time fusion of data streams from onboard navigation sensors into a condition monitoring system is yet to be seen in commercial navigation systems. Although the potential resiliency arising from the fusion of multiple sensors can be vouched, there is no formal analysis showing the concrete possibility of diagnosing cyber-attacks in a marine navigation system.

Cyber-attacks and faults are inherently different, however they produce the same effects onto the navigation system, i.e. information for navigation becomes either unavailable or untrustworthy. Under the action of a cyber-attack or the occurrence of a fault the signals and systems of interest alter their behaviour from what is the ground truth, i.e. the normal behaviour. The approach in this paper is to consider the inconsistencies of information that arise as effect of incidental defects or cyber-attack. Hence, fault diagnosis methods could be adopted to perform a systemwide analysis of consistency of information when different navigation sensors are integrated. Fault-tolerance of marine navigation systems solely including proprioceptive sensors (GNSS, IMU, speed log, compass, rate gyro) subject to faults has been addressed in [15], [16], [17], [18] where fault diagnosis, resilient sensor fusion and fault-tolerant control were in focus.

In the present study the cyber-resiliency is assessed for 


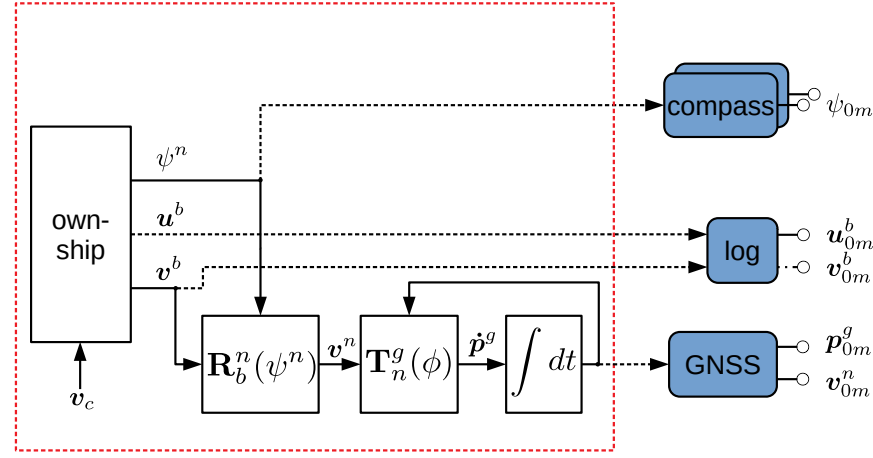

(a) The red box encapsulates own-ship's kinematics. The blue rectangles show measured quantities.

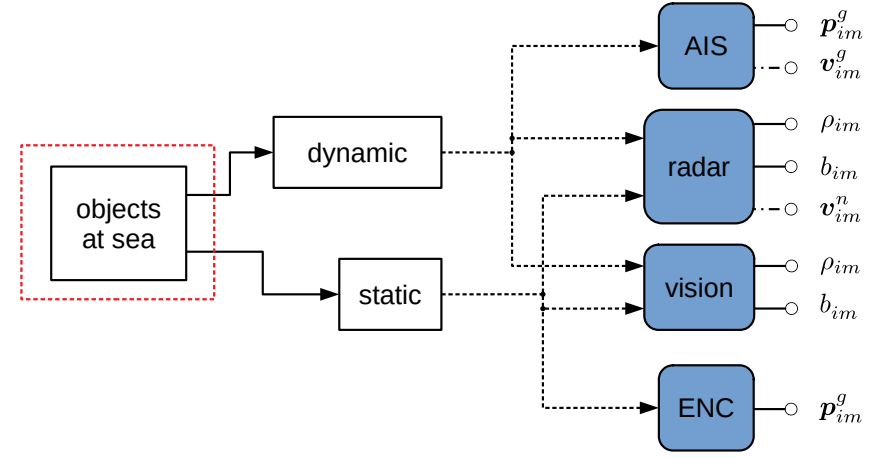

(b) Own-ship sensing of objects at sea, red box encapsulating the not shown internal kinematics.

Fig. 1: Diagrams of interaction between sensing systems and the environment. Note, dotted black lines represent measured quantities.

required marine navigation instruments according to the International Maritime Organization's (IMO) Safety of Life at Sea (SOLAS) [20], covering both own ship and observed objects. Scenarios of coastal water navigation characterized by different combinations of static (e.g. buoys and landmarks) and dynamic (e.g. vessels) objects in the surrounding of own ship are considered. Further, sensor information sourced from commercially available high-level instrument interfaces is considered as opposed to raw measurements, which are likely hidden behind proprietary protocols. The analysis employs signal paths and system topology to determine behavioural relations among measured quantities; then the structural analysis (see [19] and references herein) is adopted to assess detectability and isolability properties of the whole navigation system under the occurrence of multiple malfunctions, including GNSS and AIS. The paper illustrates how residual signals generated from simulated data are used to validate the results, and shows that all single and several cases of simultaneous defects are discovered. The results demonstrate that resilience of navigation information can be obtained for navigation in coastal waters.

\section{System DesCRIPTION}

The system of interest is a surface vessel travelling in coastal waters, and equipped with the IMO SOLAS required navigation sensors [20]. These include GNSS receiver, speed log, compass, radar, Echo Sounder to measure depth to sea floor, Electronic Navigation Chart (ENC) and Automatic Identification System (AIS) transponder. Our analysis extends this set by a computer-aided vision system that detects and classify surrounding objects to provide situational awareness. Figures $1 \mathrm{a}-1 \mathrm{~b}$ provide a visualization of how the sensors probe the pose of own ship and other objects at sea.

The following sections consider navigation scenarios wherein there exists several objects. Static objects according to the sea chart and dynamic ones like vessels at sea. A variable with the subscript 0 pertains to the own ship. With other objects present, variables pertaining to these will have consecutive subscripts $i$.

\section{A. Reference Frames}

Sensor signals are described with respect to well-known reference frames. This is done using superscripts to indicate the frame in which a quantity is measured or represented. These are: $\{\mathrm{g}\}$ for geodetic reference frame; $\{\mathrm{e}\}$ for Earth-centred Earth-fixed (ECEF) rectangular frame; $\{\mathrm{n}\}$ for North-East-Down (NED) frame; $\{b\}$ for body-fixed manoeuvring coordinates (forward, starboard, down).

A geographical position is $\boldsymbol{p}^{g}=[\phi, \lambda, h]^{T}$, defined by latitude $\phi$, longitude $\lambda$ and altitude $h$. A position in the ECEF rectangular frame is given by $\mathbf{p}^{e}=\left[x^{e}, y^{e}, z^{e}\right]^{T}$. A position on the NED frame is $\boldsymbol{p}^{n}=[N, E, D]^{T}$. The body frame is vessel-fixed, with origin at midships, waterline.

Coordinate frame transformations are listed in the following; for an more in-depth treatment see e.g. [21, Chapter 2].

1) Position in Geodetic Frame to ECEF Rectangular Frame: Let $\mathbf{T}_{g}^{e}$ be the transformation that maps the position expressed in ECEF geodetic to ECEF rectangular frame, according to the definition from [21, Sec. 2.3.3]. Then the transformation of position vectors is formulated as

$$
\boldsymbol{p}^{e}=\mathbf{T}_{g}^{e}\left(\boldsymbol{p}^{g}\right)
$$

2) Velocity in NED Frame to Geodetic Frame: Let the transformation for the velocity vector expressed in NED frame to geodetic frame be given by $\mathbf{T}_{n}^{g}$ following the definition from [21, Sec. 2.5.3]. As such the transformation of velocity vectors is formulated as

$$
\dot{\boldsymbol{p}}^{g}=\mathbf{T}_{n}^{g}\left(\boldsymbol{p}^{g}\right) \boldsymbol{v}^{n}
$$

where $\boldsymbol{v}^{n}$ is the velocity vecotr in the $\{\mathrm{n}\}$-frame. The inverse relationship exists and is given by

$$
\boldsymbol{v}^{n}=\mathbf{T}_{g}^{n}\left(\boldsymbol{p}^{g}\right) \dot{\boldsymbol{p}}^{g}
$$

3) Velocity in NED Frame to Body-fixed Frame: Let $\mathbf{R}_{b}^{n}\left(\Theta_{n b}\right)$ be the orthonormal rotation matrix from bodyfixed to NED frame given as a function of the Euler angles vector $\Theta_{n b}$ [22, Sec. 2.2.1]. Therefore, the transformation of velocity vectors is given by

$$
\boldsymbol{v}^{b}=\mathbf{R}_{n}^{b}\left(\Theta_{n b}\right) \boldsymbol{v}^{n}
$$


where $\mathbf{R}_{n}^{b}=\left(\mathbf{R}_{b}^{n}\right)^{T}$. For navigation of surface vessels the motion is studied in the horizontal plane, therefore the rotation from $\{\mathrm{n}\}$-frame to $\{\mathrm{b}\}$-frame is given by $\mathbf{R}_{n}^{b}(\psi)$, where $\psi$ is the ship's heading angle.

\section{B. Measurement Sources}

This section introduces the different measurement sources that are taken into consideration in this study as well as modelling assumptions regarding these measuring devices.

1) GNSS: The GNSS provides measurements of the geodetic position, speed and course over ground (SOG, $\mathrm{COG}$ ). SOG and $\mathrm{COG}$ are derived as a function of the geodetic position.

2) Compass: The gyro compass provides measurements of the ship's heading $\psi^{n}$. In line with IMO SOLAS [20], it is assumed that the own-ship has a total of two independent compass sensor units.

3) Speed log: The speed log provides measurements of the speed through water (STW) in longitudinal and lateral directions, given by $\boldsymbol{u}^{b}$. Given sufficiently shallow water, it also measures velocity with respect to ground $\boldsymbol{v}^{b}$. Since the paper considers vessels sailing in coastal waters, the availability of SOG and COG through the speed $\log$ is considered realistic but not guaranteed.

4) Radar system: The radar system provides measurements of range $\rho$ and bearing $b$ between the own ship and object $i$, which are subsequently used to provide estimates of SOG and COG. The range is the straight line distance between own ship and a target. The bearing is the angle between the heading of the own ship and the line-of-sight to the target. The velocity estimate is given with respect to the NED frame aligned with the own ship's body-fixed frame, as such for velocity in $\{\mathrm{n}\}$-frame one can apply the rotation matrix $\mathbf{R}_{b}^{n}(\psi)$. The marine radar system being considered here provides a $360^{\circ}$ field-of-view.

5) Vision system: The vision system is assumed to consist of multiple cameras operating at different wavelengths, and a processing unit that can detect and classify objects present in the images by exploiting advanced computer vision algorithms. Based on such detections, it then provides measurements of range and bearing between the own ship and object $i$. It is assumed that the vision system has a $360^{\circ}$ field-of-view, and lower range than the radar system.

6) AIS: The AIS system provides information about the latitude, longitude, SOG and COG of other vessels.

7) ENC: The ENC can be referenced to provide information regarding the latitude and longitude of static objects like buoys, lighthouses, etc. It is assumed that objects detected in the radar and vision systems have been correlated with the corresponding object in the ENC, if applicable.

\section{Definitions}

For a surface vessel navigating in coastal waters, let $\mathcal{O}$ be the set of all identifiable objects in the range of the radar system. This set is defined as the union of static, $\mathcal{O}_{S}$, and dynamic objects, $\mathcal{O}_{D}$, such that

$$
\mathcal{O}=\mathcal{O}_{S} \cup \mathcal{O}_{D}
$$

Here, static means objects at a fixed position. A buoy moored to the sea bed and a lighthouse on land are static objects, while ships are dynamic objects.

Every identifiable object, $o_{i} \in \mathcal{O}$, is thus parametrized as

$$
o_{i}=\left\{\boldsymbol{p}_{i}^{g}, \rho_{i}, b_{i}, \boldsymbol{v}_{i}^{n}\right\}
$$

where for static objects it is assumed that $\boldsymbol{v}^{n}=0$. These object parameters are then measured or estimated by the different sensor systems.

1) Radar: Let the set of objects identified by the radar system $\mathcal{R}$ be given as the subset of identifiable objects

$$
\mathcal{R} \subset \mathcal{O}
$$

where an object detected by the radar, $o_{i}^{r} \in \mathcal{R}$ is described by range, bearing, and a velocity as

$$
o_{i}^{r}=\left\{\rho_{i}, b_{i}, \boldsymbol{v}_{i}^{n}\right\}
$$

2) Vision: Let the set of objects identified by the vision system $\mathcal{V}$ be given as the subset of identifiable objects

$$
\mathcal{V} \subset \mathcal{O}
$$

where an object detected by the vision, $o_{i}^{v} \in \mathcal{V}$ is described by range and bearing as

$$
o_{i}^{v}=\left\{\rho_{i}, b_{i}\right\}
$$

Note that objects detected by the radar are not necessarily detected by the vision system, e.g. due to difference in measurement range. Likewise, not all objects detected by vision are necessarily detected by radar. This includes leisure boats as kayaks with low radar cross section. Therefore, $\mathcal{R} \cap \mathcal{V} \subseteq \mathcal{R} \cup \mathcal{V}$

3) ENC: The set of objects reported in the ENC $\mathcal{E}$ is a subset of the set of static objects, i.e. $\mathcal{E} \subset \mathcal{O}_{S}$. A static object in the $\mathcal{E}$ set is parametrized as

$$
e_{i}=\left\{\boldsymbol{p}_{i}^{g}\right\}
$$

4) AIS: The set of objects $\mathcal{A}$ identified through AIS messages is a subset of the set of dynamic objects, i.e. $\mathcal{A} \subset \mathcal{O}_{D}$. A dynamic object in $\mathcal{A}$ is parametrized as

$$
a_{i}=\left\{\boldsymbol{p}_{i}^{g}, \boldsymbol{v}_{i}^{n}\right\}
$$

Note that the set of identifiable objects $\mathcal{O}$ is then the result of the union of the radar, vision, ENC and AIS objects sets, i.e.

$$
\mathcal{O}=\mathcal{R} \cup \mathcal{V} \cup \mathcal{E} \cup \mathcal{A}
$$

\section{Mathematical Relationships}

Fundamental mathematical relationships existing among various measured quantities are now introduced and discussed. These will be instrumental when performing the cyber-resilience analysis exploiting behavioural relations and structural analysis. 
1) Distance: The distance between own ship and object $i$ is the 2-norm of the difference of the two ECEF rectangular coordinates

$$
\operatorname{dist}\left(\boldsymbol{p}_{0}^{e}, \boldsymbol{p}_{i}^{e}\right)=\left\|\boldsymbol{p}_{0}^{e}-\boldsymbol{p}_{i}^{e}\right\|_{2}
$$

Applying the relevant transformation this can be phrased with respect to geodetic coordinates

$$
\operatorname{dist}\left(\boldsymbol{p}_{0}^{e}, \boldsymbol{p}_{i}^{e}\right)=\left\|\mathbf{T}_{g}^{e}\left(\boldsymbol{p}_{0}^{g}\right)-\mathbf{T}_{g}^{e}\left(\boldsymbol{p}_{i}^{g}\right)\right\|_{2}
$$

2) Bearing: The bearing of an object with respect to ownship is computed from the geographic positions as,

$$
\operatorname{bear}\left(\boldsymbol{p}_{0}^{g}, \boldsymbol{p}_{i}^{g}, \psi_{0}\right)=\operatorname{atan} 2\left(\bar{c}_{i}, \underline{c}_{i}\right)-\psi_{0}
$$

where

$$
\begin{aligned}
\bar{c}_{i} & =\sin (\Delta \lambda) \cos \left(\phi_{i}\right) \\
\underline{c}_{i} & =\cos \left(\phi_{0}\right) \sin \left(\phi_{i}\right)-\sin \left(\phi_{0}\right) \cos \left(\phi_{i}\right) \cos (\Delta \lambda)
\end{aligned}
$$

and $\Delta \lambda=\lambda_{i}-\lambda_{0}$.

3) Velocity in NED frame from GNSS: For many vessels the GNSS is used for measurements of COG and SOG, in other words the NED frame velocity vector. This means that the malfunction of the GNSS unit should affect both the position and velocity measurements. This correlation can be enforced by modelling the position as a measurement and the velocity as a constraint equation.

The calculation for $\{\mathrm{n}\}$-frame velocity from $\{\mathrm{g}\}$-frame position is made by differentiating the position and applying a $\{\mathrm{g}\}$ to $\{\mathrm{n}\}$ transformation, as in (2).

4) Side-slip angle: When sailing in the presence of sea currents the difference between COG and heading is the sideslip angle $\beta$ and given as [22, Definition 2.6],

$$
\chi=\psi+\beta
$$

where the COG, $\chi$, is the direction of the velocity over ground vector $\operatorname{ang}\left(\boldsymbol{v}^{n}\right)$. The side-slip angle is only defined when the ship's forward speed $U \neq 0$ [22, Eq. 2.98]

$$
\beta=\sin ^{-1}\left(\frac{v_{r}}{U}\right)
$$

where $v_{r}$ is the lateral component of the relative velocity vector $\boldsymbol{u}^{b}$ of a craft expressed in body frame. This can be equivalently written as the angle of the $\{b\}$-frame relative velocity vector $\boldsymbol{u}^{b}$

$$
\beta=\operatorname{ang}\left(\boldsymbol{u}^{b}\right)
$$

5) Velocity determined from static landmarks: Given range and bearing measurements for an individual static landmark as well as heading for the own ship one can generate an estimate for the $\{n\}$-frame velocity of own ship. This is convenient for interlinking the own ship dynamics with objects found by the radar and vision systems.

The position of the own ship in $\{n\}$-frame can be found by describing the ship in the $\psi_{0}$ rotated landmark body-fixed frame and then by applying the corresponding $\{b\}$ to $\{n\}$ rotation such that

$$
\boldsymbol{p}_{0}^{n}=\boldsymbol{p}_{i}^{n}+\mathbf{R}_{b}^{n}\left(\psi_{0}^{n}\right) \rho_{i}\left[\begin{array}{l}
-\cos \left(b_{i}\right) \\
-\sin \left(b_{i}\right)
\end{array}\right]
$$

for stationary object $i$.

The $\{n\}$-frame velocity can thus be found by differentiation

$$
\begin{aligned}
\boldsymbol{v}_{0}^{n}= & \left(\dot{\mathbf{R}}_{b}^{n}\left(\psi_{0}^{n}\right) \rho_{i}+\mathbf{R}_{b}^{n}\left(\psi_{0}^{n}\right) \dot{\rho}_{i}\right)\left[\begin{array}{l}
-\cos \left(b_{i}\right) \\
-\sin \left(b_{i}\right)
\end{array}\right] \\
& +\mathbf{R}_{b}^{n}\left(\psi_{0}^{n}\right) \rho_{i} \dot{b}_{i}\left[\begin{array}{c}
\sin \left(b_{i}\right) \\
-\cos \left(b_{i}\right)
\end{array}\right]
\end{aligned}
$$

This assumes that $\dot{\boldsymbol{p}}_{i}^{n}=0$, which is true for a static object.

\section{FRAMEWORK FOR ANALYSIS OF CYBER-RESILIENCE}

The framework for analysis of the cyber-resilience of the marine navigation system consists of the derivation of behavioural relations (i.e. constraints) linking physical quantities directly measured or derived from measurements, and the adoption of structural analysis to identify which of such relations can be exploited for detection and isolation of cyber-attacks. The behaviours listed in Table I include all instruments required by IMO for safe navigation. The set of normal behaviours is therefore complete. A salient feature of the analysis based on topology is that, given that the basic set of normal behaviours is complete, then the analysis is provable correct, and the conclusions about detecting deviations from normal behaviours are also complete.

The basic procedure of a structural analysis is well known [19], therefore it is only briefly summarized. First, a set of constraints is formulated that describe the set of normal behaviours. Second, the structure of the system is formulated as a system graph and an equivalent incidence matrix. These express which unknown variables enter in which constraints. Third, graph theory methods are used to determine how all unknown variables can be calculated (matched). The matching describes paths in the system graph that shows how unknown variables are calculated from input and measurements. Constraints that are not used in a matching are used as redundancy relations. Inserting the unknown variables in the unmatched constraints and using the paths leading back to known variables (backtracking), turns unmatched constraints into residual signals that are used for diagnosis. Each residual signal is sensitive to violation of constraints that take part in its calculation. This means that a violation of any constraint that is participating in the calculation of a residual are structurally detectable. With a set of residual signals available, violations of constraints can be structurally isolable. Analysis of isolability properties is of particular importance since this property enable us to isolate which sensor or subsystem has been compromised or has an accidental defect.

Violation of behavioural relations indicates that physical constraints between quantities are not verified by a current set of measurements. Such violation could be caused by a physical fault or by malicious tampering. This is indeed the strength of structural analysis, which allows a systematic review of the system resiliency through a framework that unifies faults and cyber-attacks by analysing the system through the lens of functional availability. 
TABLE I: Behavioural relations used by Structural Analysis.

\begin{tabular}{|c|c|c|}
\hline Type & Equation & \\
\hline$c_{1}$ & $\operatorname{ang}\left(\boldsymbol{v}_{0}^{n}\right)=\psi_{0}^{n}+\beta$ & \\
\hline$c_{2}$ & $\beta=\operatorname{ang}\left(\boldsymbol{u}_{0}^{b}\right)$ & \\
\hline$c_{3}$ & $\boldsymbol{u}_{0}^{n}=\boldsymbol{v}_{0}^{n}-\boldsymbol{v}_{c}^{n}$ & \\
\hline$c_{4}$ & $\boldsymbol{u}_{0}^{n}=\mathbf{R}_{b}^{n}\left(\psi_{0}^{n}\right) \boldsymbol{u}_{0}^{b}$ & \\
\hline$c_{5}$ & $\boldsymbol{v}_{0}^{n}=\mathbf{R}_{b}^{n}\left(\psi_{0}^{n}\right) \boldsymbol{v}_{0}^{b} \quad$ if shallow water & \\
\hline$c_{6}$ & $\dot{\boldsymbol{p}}_{0}^{g}=\mathbf{T}_{n}^{g}\left(\boldsymbol{p}_{0}^{g}\right) \boldsymbol{v}_{0}^{n}$ & \\
\hline$c_{7, i}$ & $\rho_{i}=\operatorname{dist}\left(\boldsymbol{p}_{0}^{g}, \boldsymbol{p}_{i}^{g}\right) \quad$ for $i \in \mathcal{O}$ & \\
\hline$c_{8, i}$ & $b_{i}=\operatorname{bear}\left(\boldsymbol{p}_{0}^{g}, \boldsymbol{p}_{i}^{g}, \psi_{0}^{n}\right) \quad$ for $i \in \mathcal{O}$ & \\
\hline$c_{9, i}$ & $\boldsymbol{v}_{0}^{n}=\frac{d}{d t}\left(\mathbf{R}_{b}^{n}\left(\psi_{0}^{n}\right) \rho_{i}\left[\begin{array}{l}-\cos \left(b_{i}\right) \\
-\sin \left(b_{i}\right)\end{array}\right]\right)$ & for $i \in \mathcal{O}_{S}$ \\
\hline$c_{10, i}$ & $\boldsymbol{v}_{i}^{n}=\mathbf{R}_{b}^{n}\left(\psi_{0}^{n}\right) \boldsymbol{v}_{i}^{b} \quad$ for $i \in \mathcal{O}_{D}$ & \\
\hline$c_{11, i}$ & $\dot{\boldsymbol{p}}_{i}^{g}=\mathbf{T}_{n}^{g}\left(\boldsymbol{p}_{i}^{g}\right) \boldsymbol{v}_{i}^{n} \quad$ for $i \in \mathcal{O}_{D}$ & \\
\hline$d_{1}$ & $\dot{\boldsymbol{p}}_{0}^{g}=\frac{d}{d t} \boldsymbol{p}_{0}^{g}$ & \\
\hline$d_{2, i}$ & $\dot{\rho}_{i}=\frac{d}{d t} \rho_{i} \quad$ for $i \in \mathcal{O}_{S}$ & \\
\hline$d_{3, i}$ & $\dot{b}_{i}=\frac{d}{d t} b_{i} \quad$ for $i \in \mathcal{O}_{S}$ & \\
\hline$d_{4, i}$ & $\dot{\boldsymbol{p}}_{i}^{g}=\frac{d}{d t} \boldsymbol{p}_{i}^{g} \quad$ for $i \in \mathcal{O}_{D}$ & \\
\hline$m_{1}$ & $\boldsymbol{p}_{0 m}^{g n s s}=\boldsymbol{p}_{0}^{g}$ & \\
\hline$m_{2}$ & $\psi_{0 m 1}^{c m p}=\psi_{0}^{n}$ & \\
\hline$m_{3}$ & $\psi_{0 m 2}^{c m p}=\psi_{0}^{n}$ & \\
\hline$m_{4}$ & $\boldsymbol{u}_{0 m}^{\log }=\boldsymbol{u}_{0}^{b}$ & \\
\hline$m_{5}$ & $\boldsymbol{v}_{0 m}^{l o g}=\boldsymbol{v}_{0}^{b} \quad$ if shallow water & \\
\hline$m_{6, i}$ & $\rho_{i m}^{\text {radar }}=\rho_{i} \quad$ for $i \in \mathcal{R}$ & \\
\hline$m_{7, i}$ & $b_{i m}^{\text {radar }}=b_{i} \quad$ for $i \in \mathcal{R}$ & \\
\hline$m_{8, i}$ & $\boldsymbol{p}_{i m}^{e n c}=\boldsymbol{p}_{i}^{g} \quad$ for $i \in \mathcal{E}$ & \\
\hline$m_{9, i}$ & $\boldsymbol{p}_{i m}^{a i s}=\boldsymbol{p}_{i}^{g} \quad$ for $i \in \mathcal{A}$ & \\
\hline$m_{10, i}$ & $\boldsymbol{v}_{i m}^{a i s}=\boldsymbol{v}_{i}^{n} \quad$ for $i \in \mathcal{A}$ & \\
\hline$m_{11, i}$ & $\boldsymbol{v}_{i m}^{\text {radar }}=\boldsymbol{v}_{i}^{b} \quad$ for $i \in \mathcal{O}_{D} \cap \mathcal{R}$ & \\
\hline$m_{12, i}$ & $\rho_{i m}^{\text {vision }}=\rho_{i} \quad$ for $i \in \mathcal{V}$ & \\
\hline$m_{13, i}$ & $b_{i m}^{\text {vision }}=b_{i} \quad$ for $i \in \mathcal{V}$ & \\
\hline
\end{tabular}

A complete set of the behavioural relations that describe the marine navigation systems is presented in Table I. The constraints $c_{1}-c_{11, i}$ are mathematical relations derived from the vessel kinematics as well as using the transformation of coordinates introduced in Section II; $d_{1}-d_{4, i}$ are differential constraints; $m_{1}-m_{13, i}$ are measurement constraints linking mathematical variables defined in $c_{1}-c_{11, i}$ with physical quantities measured by the sensors. The constraints $c_{i}$ and $d_{j}$ cannot be violated since they express mathematical relations between measured variables.

\section{Diagnosis of COMPRomised or Defect SUBSYSTEMS}

Using the complete description of normal behaviours from Table I, efficient tools are available to analyse system topology (structure) and finds all minimally structurally overdetermined (MSO) sets [23]. Detectability and isolability is obtained from analysis of the signature that violations give rise to in a set of residuals [19]. The result is an isolability matrix that for each residual lists which sensor system defects are structurally detectable from individual residuals, and isolable using a combination of residuals. This is illustrated in Table II. Algorithms exist that can select combinations of residuals to optimize isolability [24]. Applying these is an essential step for implementation, but was considered out of scope for the present paper.

For the navigation system, some constraints are conditional based on which external objects are in range of the sensors. This implies that the set of behavioural relation has variable cardinality, and therefore a set of residuals that vary in number (instances) as a consequence of the number of objects in range. This is explored in the following by analysing few operational scenarios for a vessel sailing in coastal waters. The following subsections exemplify scenarios and also address the case of more than one sensor subsystem being compromised or accidentally failed.

Case 1 - SOLAS sensors: This is the general case scenario for coastal navigation of a marine vessel containing all required sensors as per the IMO SOLAS convention. It is assumed that the water is sufficiently shallow for measurements of SOG and COG from the DVL. Assume also that in this local area there is one static and one dynamic object, both of which are identifiable by the radar system, such that $\exists o_{1}^{r} \in \mathcal{O}_{S}, \exists o_{2}^{r} \in \mathcal{O}_{D} \Rightarrow|\mathcal{R}|=2$.

Case 2 - SOLAS sensors plus vision: This is a proposed modification to the general scenario Case 1. The idea is that augmenting the onboard sensing system with a vision system will enable increased isolability during multi-fault scenarios. This results in having the same two objects from Case 1 being identified by both radar and vision systems, such that $\exists o_{1}^{r} \in \mathcal{O}_{S}, \exists o_{2}^{r} \in \mathcal{O}_{D} \Rightarrow|\mathcal{R}|=2$ and $\exists o_{1}^{v} \in \mathcal{O}_{S}, \exists o_{2}^{v} \in$ $\mathcal{O}_{D} \Rightarrow|\mathcal{V}|=2$. This scenario is analysed for multiple faults in GNSS and AIS systems.

Case 3 - Simplified: This is a simplified version of Case 1 , with the intent of presenting some numerical simulation results. It is proposed that in this scenario the vessel in question utilizes standard navigation sensors. Assume that the water is sufficiently shallow for measurements of SOG and COG from the DVL, in addition assume that in the proximity of the vessel there exists a single static object which is identifiable from the radar, such that $\exists o_{1}^{r} \in \mathcal{O}_{S} \Rightarrow$ $|\mathcal{R}|=1$. Figure 2 illustrates the simulated scenario.

Applying the MSO matching algorithm [23] to the resulting subset of constraints results in 47 each of matchings and parity equations. Using the latter, one can make an isolability analysis on the constraints; for this system the following subset of parity equations

$$
\begin{aligned}
& r_{1}=\psi_{0 m 1}^{c m p}-\psi_{0 m 2}^{c m p} \\
& r_{2}=b_{1 m}^{r a d a r}-\operatorname{bear}\left(\boldsymbol{p}_{0 m}^{g n s s}, \boldsymbol{p}_{1 m}^{e n c}, \psi_{0 m 2}^{c m p}\right) \\
& r_{3}=b_{1 m}^{\text {radar }}-\operatorname{bear}\left(\boldsymbol{p}_{0 m}^{g n s s}, \boldsymbol{p}_{1 m}^{e n c}, \psi_{0 m 1}^{c m p}\right) \\
& r_{4}=\rho_{1 m}^{r a d a r}-\operatorname{dist}\left(\boldsymbol{p}_{0 m}^{g n s s}, \boldsymbol{p}_{1 m}^{e n c}\right) \\
& r_{5}=\mathbf{T}_{n}^{g}\left(\boldsymbol{p}_{0 m}^{\text {gnss }}\right)\left[\frac{d}{d t}\left(\mathbf{R}_{b}^{n}\left(\psi_{0 m 2}^{c m p}\right) \rho_{1 m}^{\text {radar }}\left[\begin{array}{c}
-\cos \left(b_{1 m}^{\text {radar }}\right) \\
\sin \left(b_{1 m}^{\text {radar }}\right)
\end{array}\right]\right)\right] \\
& -\frac{d}{d t} \boldsymbol{p}_{0 m}^{g n s s}
\end{aligned}
$$




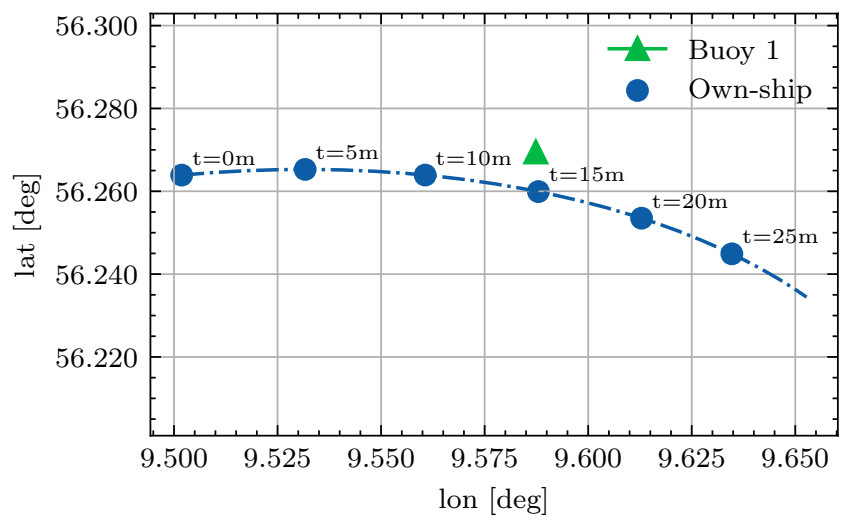

Fig. 2: Simulated scenario utilized to demonstrate the detection framework for Case 3 - Simplified.

TABLE II: Detectability of sensor system defects in individual residuals and isolability for the entire set of residuals. For brevity, the table focus on the constraints for Case 1.

\begin{tabular}{ccccccccc}
\hline & $m_{1}$ & $m_{2}$ & $m_{3}$ & $m_{4}$ & $m_{5}$ & $m_{6,1}$ & $m_{7,1}$ & $m_{8,1}$ \\
\hline $\mathbf{1}$ & & $d$ & $d$ & & & & & \\
$\mathbf{2}$ & $d$ & & $d$ & & & & $d$ & $d$ \\
$\mathbf{3}$ & $d$ & $d$ & & & & $d$ & $d$ & $d$ \\
$\mathbf{4}$ & $d$ & & & & & $d$ & $d$ & $d$ \\
$\mathbf{5}$ & $d$ & & $d$ & & & & & \\
$\vdots$ & & & & & & & & \\
$\mathbf{2 1}$ & $d$ & & $d$ & & $d$ & & & \\
$\vdots$ & & & & & & & & \\
$\mathbf{3 4}$ & $d$ & $d$ & & $d$ & & & & \\
$\vdots$ & & & & & & & & \\
\hline ALL & $i$ & $i$ & $i$ & $i$ & $i$ & $i$ & $i$ & $i$ \\
\hline
\end{tabular}

$$
\begin{aligned}
r_{21}= & \mathbf{R}_{b}^{n}\left(\psi_{0 m 2}^{c m p}\right) \mathbf{T}_{n}^{g}\left(\boldsymbol{p}_{0}^{g n s s}\right) \boldsymbol{v}_{0 m}^{l o g}-\frac{d}{d t} \boldsymbol{p}_{0 m}^{g n s s} \\
r_{34}= & \operatorname{ang}\left(\mathbf{T}_{g}^{n}\left(\boldsymbol{p}_{0 m}^{g n s s}\right) \frac{d}{d t} \boldsymbol{p}_{0 m}^{g n s s}\right)-\operatorname{ang}\left(\boldsymbol{u}_{0 m}^{\log }\right) \\
& -\psi_{0 m 1}^{c m p}
\end{aligned}
$$

suffices to obtain full isolability, see Table II where $d$ denotes detectable; $i$ stands for isolable. This means that if the residual $r_{1}$ triggers then is either $m_{2}$ or $m_{3}$ being violated, but if residuals $r_{2}$ though $r_{5}$ trigger then it must be $m_{1}$ that is violated. This behaviour is confirmed after inspecting the residual time-series corresponding to (25)-(30) in Fig. 3 of the simulated Case 3 in Fig. 2.

\section{A. Implementation}

In the implementation of the diagnostic system, all residuals will be evaluated in parallel, and conclusions about single or multiple defects will be made based on the overall pattern. When the number of objects in view change, instances of residuals are changed accordingly. Mechanisms to obtain such dynamic behaviour were outlined in [17].

\section{Single And Multiple Violations Scenarios}

If the navigation system of own ship is fully functional, then each scenario described in Section III attains full isolability for every behavioural relation that can be violated, which in this case means measurement constraints.

Now assume that a malfunction in the GNSS has been isolated and the measurements from the GNSS have thus been discarded as invalid. This reduces the overall isolability. Table III shows the effect of an identified GNSS malfunctioning on the overall isolability of every other measurement in the scenarios introduced in Section III. Here it can be seen that the inclusion of additional sensing systems, such as the vision system, greatly improves the isolability by providing much needed redundant measurements during the GNSS outage.

Last, the scenario when multiple sensors are malfunctioning at the same time is investigated, and results of system resiliency are shown in Table IV. Here it assumed that either parts of or the entire AIS system is compromised simultaneously with the GNSS. This results in reduced isolability for measurements of the dynamic objects, particularly those of position and velocity.

Note, for both tables, that detectable constraints are denoted by $d\{\#\}$ where \# is the subset it belongs to. For example, if $\left\{m_{1}, m_{2}\right\}$ are detectable but not isolable from each other they could both be assigned $d\{1\}$ whereas the likewise detectable but not isolable set $\left\{m_{3}, m_{4}\right\}$ could be given by $d\{2\}$.

\section{Conclusions}

Structural analysis was applied to address the problem of diagnosing violation of normal behaviours of navigation instruments of a marine vessel, caused by cyber-attacks or instrument defects. The paper showed how structural analysis of behaviours enabled the detection of differences between actual and measured quantities, wherein said discrepancies are provoked by cyber-attacks or occurs for technical reasons. The paper showed generic results of the analysis of behaviours that led to a generic diagnostic framework in the form of sets of residuals that will be sensitive to changes in navigation information from sensors and sea chart, and therefore be efficient to diagnose cyber-attacks or technical defects on safety-critical navigation information.

The analysis showed isolability in different scenarios that are typical during near coast passage. It was in particular shown how defects in GNSS provided position, gyro compass heading, speed log reading and AIS messages are isolated and, thereby, how adverse effects on safe navigation can be avoided if essential sensors are compromised or have technical defects. Residual signals generated from simulated data validated our claims. Showing that all single and several cases of simultaneous defects are discovered, the results demonstrated that resilience of navigation information is obtainable for vessels sailing in coastal waters. 

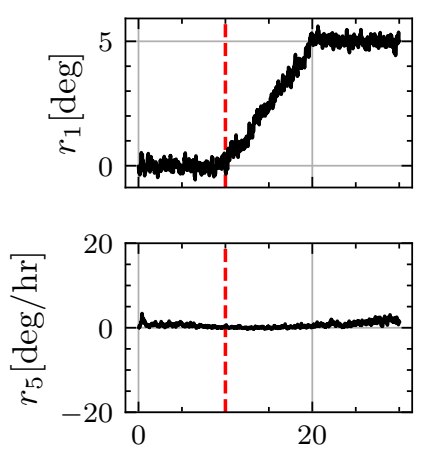

Time $[\mathrm{min}]$
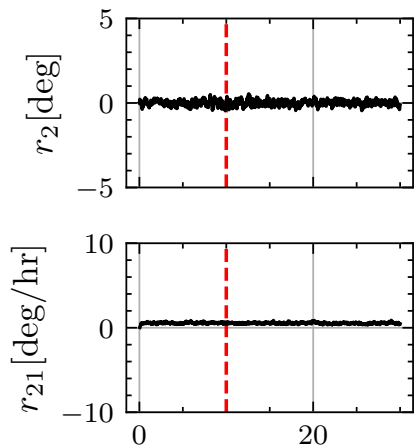

Time $[\mathrm{min}]$
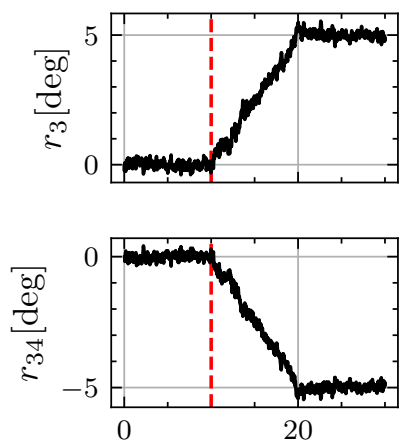

Time $[\mathrm{min}]$
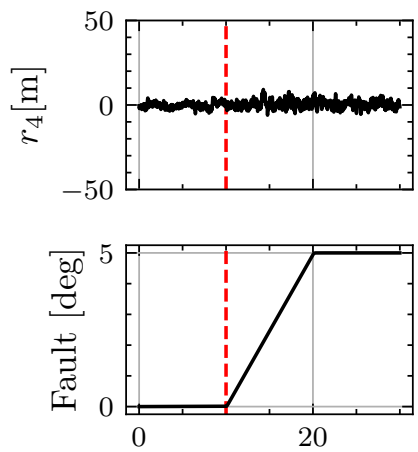

Time $[\mathrm{min}]$

Fig. 3: Time series of the residuals for the simulated scenario described in Case 3. The own ship navigates around a static landmark detectable on the radar. An incipient fault is imposed on the timestamp indicated by the vertical dashed red lines. The fault manifests as an evolving additive error in the magnetic compass readings, with rate of change of $5^{\circ}$ over $10 \mathrm{~min}$.

TABLE III: Analysis of isolability given the GNSS malfunctioning. In the table the symbol "-" means that the sensor is not included, and the symbol "0" means that the sensor cannot fail.

\begin{tabular}{|c|c|c|c|c|c|c|c|c|c|c|c|c|c|c|}
\hline \multirow{3}{*}{$\begin{array}{c}\text { Sensor } \\
\text { Scenario } \\
\end{array}$} & \multicolumn{4}{|c|}{ own-ship } & \multicolumn{4}{|c|}{$o_{1}^{s}$} & \multicolumn{6}{|c|}{$o_{2}^{d}$} \\
\hline & \multirow{2}{*}{$\begin{array}{c}\text { GNSS } \\
\boldsymbol{p}_{0}^{g} \\
\end{array}$} & compasses & \multicolumn{2}{|c|}{ Log } & \multicolumn{2}{|c|}{ Radar } & Vision & \multirow{2}{*}{$\begin{array}{c}\text { ENC } \\
p_{1}^{g} \\
\end{array}$} & \multicolumn{3}{|c|}{ Radar } & Vision & \multicolumn{2}{|c|}{ AIS } \\
\hline & & {$\left[\begin{array}{ll}\psi_{0} & \psi_{0}\end{array}\right]$} & $\boldsymbol{u}_{0}^{b}$ & $\boldsymbol{v}_{0}^{b}$ & {$\left[\rho_{1}\right.$} & $\left.b_{1}\right]$ & {$\left[\begin{array}{ll}\rho_{1} & b_{1}\end{array}\right]$} & & {$\left[\rho_{2}\right.$} & $\left.b_{2}\right]$ & $\boldsymbol{v}_{2}^{n}$ & {$\left[\begin{array}{ll}\rho_{2} & b_{2}\end{array}\right]$} & $p_{2}^{g}$ & $\boldsymbol{v}_{2}^{n}$ \\
\hline 1 & $f$ & {$\left[\begin{array}{ll}i & i\end{array}\right]$} & $i$ & $i$ & {$[i$} & $i]$ & - & 0 & {$[0$} & $0]$ & $i$ & - & $i$ & $i$ \\
\hline 2 & $f$ & {$\left[\begin{array}{ll}i & i\end{array}\right]$} & $i$ & $i$ & {$[i$} & i] & {$\left[\begin{array}{ll}i & i\end{array}\right]$} & 0 & {$[d\{1\}$} & $d\{2\}]$ & $i$ & {$[d\{1\} \quad d\{2\}]$} & $i$ & $i$ \\
\hline 3 & $f$ & {$\left[\begin{array}{ll}i & i\end{array}\right]$} & $i$ & $i$ & {$[d\{1\}$} & $d\{1\}]$ & - & 0 & . & - & - & - & - & - \\
\hline
\end{tabular}

TABLE IV: Analysis of isolability given the malfunctioning of both the GNSS and AIS.

\begin{tabular}{|c|c|c|c|c|c|c|c|c|c|c|c|c|c|c|c|}
\hline \multirow[t]{3}{*}{$f_{1}$} & \multirow[t]{2}{*}{$f_{2}$} & \multicolumn{14}{|l|}{$f_{3}$} \\
\hline & & $m_{2}$ & $m_{3}$ & $m_{4}$ & $m_{5}$ & {$\left[\begin{array}{l}m_{6,1 r} \\
m_{7,1 r}\end{array}\right]^{T}$} & {$\left[\begin{array}{l}m_{6,1 v} \\
m_{7,1 v}\end{array}\right]^{T}$} & & & & & $m_{8,1}$ & $m_{9,2}$ & $m_{10,2}$ & $m_{11,2}$ \\
\hline & - & $i$ & $i$ & $i$ & $i$ & {$\left[\begin{array}{ll}i & i\end{array}\right]$} & {$\left[\begin{array}{ll}i & i\end{array}\right]$} & {$[d\{1\}$} & $d\{2\}]$ & {$[d\{1\}$} & $d\{2\}]$ & 0 & $i$ & $i$ & $i$ \\
\hline \multirow[t]{3}{*}{$m_{1}$} & $m_{9}$ & $i$ & $i$ & $i$ & $i$ & {$\left[\begin{array}{ll}i & i\end{array}\right]$} & {$\left[\begin{array}{ll}i & i\end{array}\right]$} & {$[d\{1\}$} & $d\{2\}]$ & {$[d\{1\}$} & $d\{2\}]$ & 0 & - & $d\{3\}$ & $d\{3\}$ \\
\hline & $m_{10}$ & $i$ & $i$ & $i$ & $i$ & {$\left[\begin{array}{ll}i & i\end{array}\right]$} & {$\left[\begin{array}{ll}i & i\end{array}\right]$} & {$[d\{1\}$} & $d\{2\}]$ & {$[d\{1\}$} & $d\{2\}]$ & 0 & $d\{3\}$ & - & $d\{3\}$ \\
\hline & $\left\{\begin{array}{c}m_{9} \\
m_{10}\end{array}\right\}$ & $i$ & $i$ & $i$ & $i$ & {$\left[\begin{array}{ll}i & i\end{array}\right]$} & {$\left[\begin{array}{ll}i & i\end{array}\right]$} & {$[d\{1\}$} & $d\{2\}]$ & {$[d\{1\}$} & $d\{2\}]$ & 0 & - & - & 0 \\
\hline
\end{tabular}

\section{ACKNOWLEDGMENT}

The authors are grateful to the Danish Maritime Authority and the Danish Maritime Fund for providing the financial support to pursue this research within the SAFENAV project. Morten C. Nissov is thankful to DTU Electrical Engineering for the opportunity granted to carry out this research as part of his honour's programme within the M.Sc. in Electrical Engineering.

\section{REFERENCES}

[1] M. Balduzzi, A. Pasta, and K. Wilhoit, "A security evaluation of AIS automated identification system," in Proceedings of the 30th Annual Computer Security Applications Conference, ser. ACSAC '14. New York, NY, USA: Association for Computing Machinery, 2014, p. 436-445. [Online]. Available: https://doi.org/10.1145/2664243.2664257

[2] B. Svilicic, J. Kamahara, J. Celic, and J. Bolmsten, "Assessing ship cyber risks: a framework and case study of ECDIS security," WMU Journal of Maritime Affairs, vol. 18, no. 3, pp. 509-520, 2019.
[3] B. Svilicic, D. Brčić, S. Žuškin, and D. Kalebić, "Raising awareness on cyber security of ECDIS," TransNav: International Journal on Marine Navigation and Safety of Sea Transportation, vol. 13, no. 1, 2019.

[4] B. Svilicic, I. Rudan, V. Frančić, and D. Mohović, "Towards a cyber secure shipboard radar," Journal of Navigation, vol. 73, no. 3, p. 547-558, 2020.

[5] A. Grant, P. Williams, N. Ward, and S. Basker, "GPS jamming and the impact on maritime navigation," Journal of Navigation, vol. 62, no. 2, pp. 173-187, 2009.

[6] Q. Meng, L.-T. Hsu, B. Xu, X. Luo, and A. El-Mowafy, "A GPS spoofing generator using an open sourced vector tracking-based receiver," Sensors, vol. 19, no. 18, p. 3993, Sept. 2019.

[7] R. T. Ioannides, T. Pany, and G. Gibbons, "Known vulnerabilities of global navigation satellite systems, status, and potential mitigation techniques," Proceedings of the IEEE, vol. 104, no. 6, pp. 1174-1194, June 2016.

[8] H. Onishi, K. Yoshida, and T. Kato, "GNSS vulnerabilities and vehicle applications," in 2016 13th Workshop on Positioning, Navigation and Communications (WPNC). IEEE, Oct. 2016.

[9] Y. Liu, S. Li, Q. Fu, and Z. Liu, "Impact assessment of GNSS spoofing attacks on INS/GNSS integrated navigation system," Sensors, vol. 18, no. 5, p. 1433, May 2018. 
[10] A. J. Kerns, D. P. Shepard, J. A. Bhatti, and T. E. Humphreys, "Unmanned aircraft capture and control via GPS spoofing," Journal of Field Robotics, vol. 31, no. 4, pp. 617-636, Apr. 2014.

[11] M. L. Psiaki and T. E. Humphreys, "GNSS spoofing and detection," Proceedings of the IEEE, vol. 104, no. 6, pp. 1258-1270, June 2016.

[12] B. Kujur, S. Khanafseh, and B. Pervan, "Detecting GNSS spoofing of ADS-b equipped aircraft using INS," in 2020 IEEE/ION Position, Location and Navigation Symposium (PLANS). IEEE, Apr. 2020.

[13] D. A. Grejner-Brzezinska, C. K. Toth, T. Moore, J. F. Raquet, M. M. Miller, and A. Kealy, "Multisensor navigation systems: A remedy for GNSS vulnerabilities?" Proceedings of the IEEE, vol. 104, no. 6, pp. 1339-1353, June 2016.

[14] F. Katsilieris, P. Braca, and S. Coraluppi, "Detection of malicious AIS position spoofing by exploiting radar information," in Proceedings of the 16th International Conference on Information Fusion, FUSION 2013, 2013, pp. 1196-1203.

[15] M. Blanke, "Fault-tolerant and Diagnostic Methods for Navigation," in Proceedings of 9th International Conference on Marine Engineering Systems. Society of Naval Architects and Marine Engineers, 2003.

[16] _ , "Diagnosis and fault-tolerant control for ship station keeping," in Proceedings of the 2005 IEEE International Symposium on, Mediterrean Conference on Control and Automation Intelligent Control, 2005. IEEE, 2005.
[17] — - "Fault-tolerant Sensor Fusion for Marine Navigation," in Pro ceedings of the 7th IFAC Conference on Manoeuvring and Control of Marine Craft, 2006, pp. 1385-1390.

[18] M. Blanke and D. T. Nguyen, "Fault tolerant position-mooring control for offshore vessels," Ocean Engineering, vol. 148, pp. 426-441, Jan. 2018.

[19] M. Blanke, M. Kinnaert, J. Lunze, and M. Staroswiecki, Diagnosis and Fault-Tolerant Control. Springer Berlin Heidelberg, 2016.

[20] International Maritime Organization, "SOLAS Convention Chapter V, Regulation 19," 2002.

[21] J. Farrell, Aided navigation : GPS with high rate sensors. New York: McGraw-Hill, 2008.

[22] T. I. Fossen, Handbook of Marine Craft Hydrodynamics and Motion Control. John Wiley \& Sons, Ltd, Apr. 2011.

[23] M. Krysander, J. Aslund, and M. Nyberg, "An Efficient Algorithm for Finding Minimal Overconstrained Subsystems for Model-Based Diagnosis," IEEE Transactions on Systems, Man, and Cybernetics Part A: Systems and Humans, vol. 38, no. 1, pp. 197-206, Jan. 2008.

[24] C. Svärd, M. Nyberg, E. Frisk, and M. Krysander, "Data-driven and adaptive statistical residual evaluation for fault detection with an automotive application," Mechanical Systems and Signal Processing, vol. 45, no. 1, pp. 170-192, mar 2014. 